
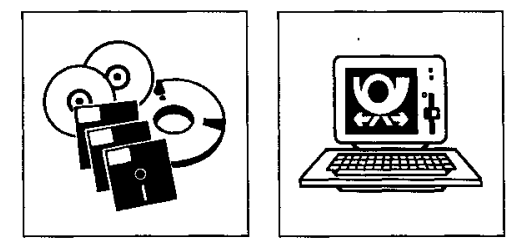

Die 'vierte Dimension' einer Datenbankrecherche

Die Online-Datenbanken des $D B I$

Kennenilernen der Datenbanken mit einem Testpaßwort

Bruno Maßfeller war Mitarbeiter am Lebrstubl für Rechtsinformatik der Universität des Saarlandes und arbeitet jetzt in der Verwaltung des Landes Brandenburg.

In jur-pc 4/92 (S. 1523) batte Mattbias Müller sich der Frage „Neue Wege der Literaturbeschaffung über Datenbanken" angenommen und skizziert, wie man Sucbergebnisse aus Datenbanken mit schneller elektronischer Bestellung der Originaldokumente kombinieren kann (und kombiniert). juris arbeitet daran, seinen Kunden diese Möglicbkeit zu bieten. Damit Juristen, die ein Urteil im Langtext benötigen, das bei juris nur im Kurztext vorbanden ist, oder die aufidie Lektïre eines Aufsatzes angewiesen sind, zu dem in der Aufsatzdatenbank nur ein Abstract vorbanden ist, auch unter Zeitdruck nicht resignieren müssen, beschreibt der folgende Beitrag, wie man beim Deutschen Bibliotheksinstitut in Berlin elektronische Bestellungen dieser Art aufgeben kann-auch zur Lieferung per Fax. (red.)

\title{
Call DBI:
}

\section{Die Datenbanken des Deutschen Bibliotheksinstituts und das 'Online document ordering'}

\section{Bruno Maßfeller}

Üblicherweise werden die Eingabe der Suchworte, deren logische Verknüpfung und die Ausgabe der auf diese Weise gefundenen Daten als die drei elementaren Rechercheoperationen in einer Datenbank genannt (vgl. nur juris Dialoghandbuch, Band 1, Auflage 1988, Seite A-17). Dies trifft aber exakt nur auf Datenbanken zu, welche das Ziel der Suche vollständig abbilden (Beispiel: Kundenkonten eines Unternehmens). Anders verhält es sich jedoch, wenn die Datenbank (ganz oder teilweise) auf eine Standortangabe und/oder eine Beschreibung ihrer Objekte beschränkt ist. Diese Art der Dokumentation wird wegen des begrenzten Speicherplatzes und aus urheberrechtlichen Gründen häufig gewählt, wenn, wie z. B. bei juris, im wesentlichen Texte erschlossen werden. Teilweise sind nur ein Abstract, ein Auszug oder eine bibliographische Beschreibung verfügbar. Demgegenüber benötigt der Benutzer oftmals den ungekürzten Text (den sog. Volltext) des Dokuments. Es wird dann als eigenständiger vierter Arbeitsschritt die Beschaffung der Dokumente notwendig. Regelmäßig ist eine weitere Suche durchzuführen, nämlich nach der Bibliothek, die das betreffende Schriftstück in ihrem Bestand führt und sowohl innerhalb des erforderlichen Zeitraums als auch der gewünschten Weise (Original, Kopie, Telefax etc.) zu liefern in der Lage ist.

Dabei kann das Deutsche Bibliotheksinstitut in Berlin mit seinen fünf Bibliothekskatalogen als Online-Datenbanken wertvolle Dienste leisten. Darüber hinaus besteht bei zwei dieser Datenbanken in gewissem, thematisch beschränktem Umfang auch die Möglichkeit der sachlichen Erschließung von Literatur. Der Schwerpunkt dieses Beitrags liegt jedoch auf dem Beschaffungsaspekt und der hierfür besonders wichtigen Zeitschriftendatenbank $\mathrm{ZDB}$.

\section{Datenbankenzugang}

Vertragliche Voraussetzungen

Zugelassen wird nur, wer als Teilnehmer registriert ist. Hierzu muß ein Aufnahmeantrag gestellt werden, mit dem die Gebühren (Stand März 1992: 40,00 DM pro verbrauchte Anschaltstunde - Grundgebühren entfallen) und die Allgemeinen Geschäftsbedingungen des DBI anerkannt werden. Daraufhin erhält jeder Benutzer eine USER-NR und ein Paßwort. Vor Abschluß eines solchen Nutzungsvertrages besteht jedoch erfreulicherweise die Möglichkeit, die Datenbanken gebührenfrei mit einem Testpaßwort unter nur geringen Einschränkungen kennenzulernen.

\section{Anwahl des DBI-Rechners und Einlogprozedur}

Wie auch sonst bei externen Datenbanken ist zwischen dem Herstellen einer Verbindung zu dem Host und der Einwahl in dessen Datenbank/en zu unterscheiden.

Das Deutsche Bibliotheksinstitut kann sowohl über das DATEX-P-Netz als auch das (nur abrechnungsmäßig, nicht leitungstechnisch hiervon zu unterscheidende) WIN $\mathbf{l} N e t z$ (Wissenschaftsnetz) erreicht werden, und zwar unter den NUA's 45300040020 bzw. 
45050130160. Der Eingang in das Datex-P-Netz verläuft normalerweise ähnlich wie in dem folgenden Downloadprotokoll:

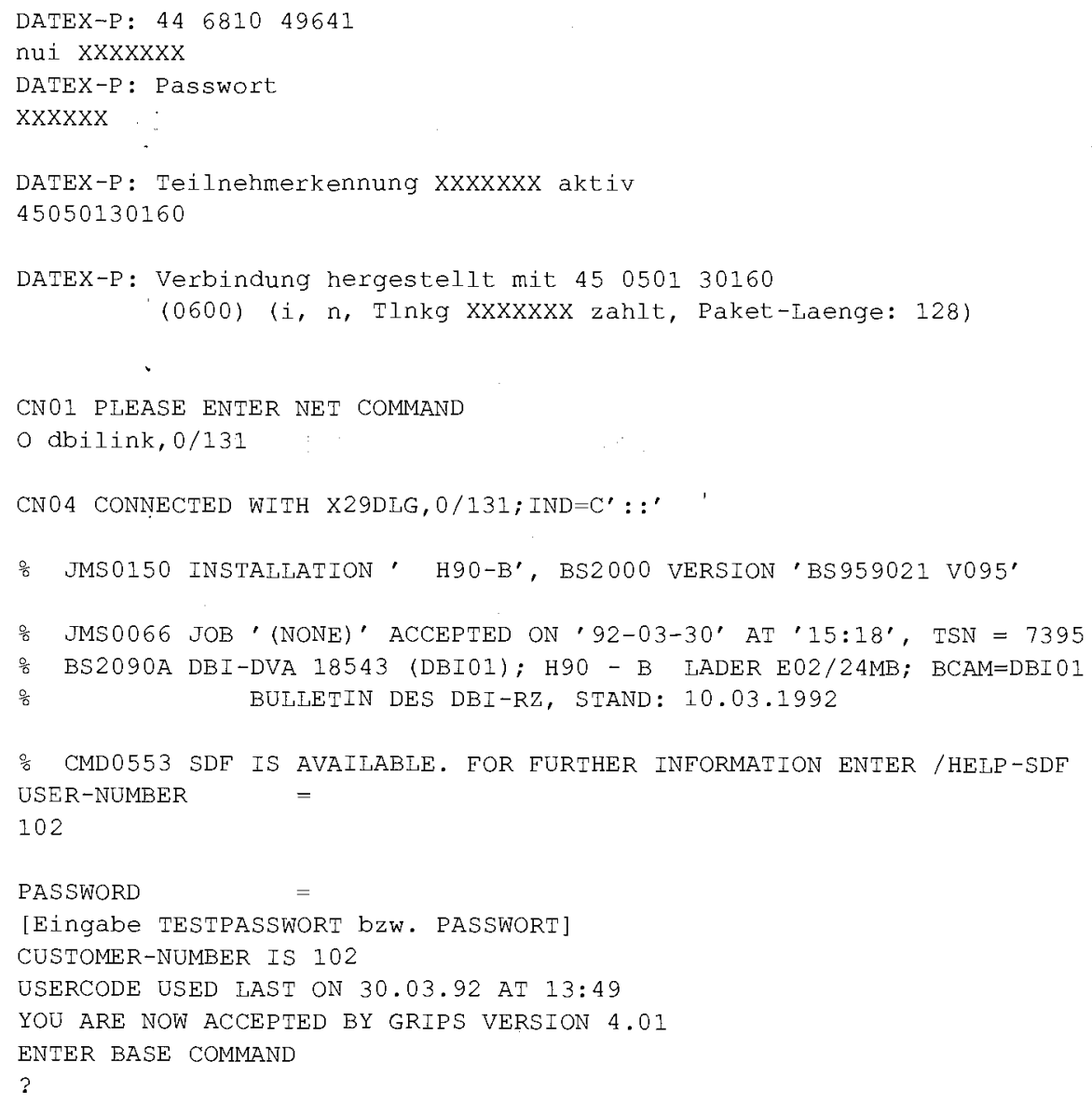

Es werden jeweils nach der Aufforderung durch das System zunächst ein NETZKOMMANDO und hiernach USERNUMMER und PASSWORD eingegeben.

Der bisher zurückgelegte Weg ist zugegebenermaßen etwas mühselig und (schreib-)fehlerträchtig, sofern man sämtliche Eingaben über die Tastatur vornimmt. Fast alle neueren Telekommumnikationsprogramme bieten aber an, derartige Dialoge über besondere Programme, sog. Scripts, zu automatisieren oder wenigstens jeweils eine Taste bzw. Tastenkombination mit den einzelnen Benutzerantworten zu „belegen”, bei deren Betätigung die gesamte Eingabe „abgeschickt” wird.

(Für jur-pc-Abonnenten ist auf.der Diskettenbeilage zu diesem Heft ein Script für das Telekommunikationsprogramm Voyager enthalten, das die automatische Einwabl in den DBIRechner übernimmt. Voyager war Bestandteil der Diskettenbeilage zum Mai-Heft. - red. -)

\section{Datenbankauswahl - Das Angebot des DBI}

Anmerkungen zum Entwicklungsstand:

Die hier wie auch an anderer Stelle in diesem Beitrag erwähnten Zahlen und Zeitangaben beruhen dabei auf dem von dem DBI herausgegebenen INFO-KURZANLEITUNG für Benutzer der Zeițchriftendatenbank - ZDB - und weiterer Datenbanken des DBI von $D$ r. Traute Braun (Stand Februar 1992) sowie den Online Informationen (Stand März 1992) und den im Mai 1993 dem Autor von dem DBI gegebenen Hinweisen auf Änderungen, die nachstehend kurz zusammengefaßt werden und soweit erforderlich in diesem Beitrag berücksichtigt wurden. Die Zeitschriftendatenbank ZDB hat nunmehr ein vierwöchentliches Update, was einen beträchtlichen Aktualitätsgewinn bedeutet. Die Zahl der Biblio- theken, die Online-Ordering anbieten, ist von 6 auf 8 angewachsen. Die größten Änderungen weist der Verbundkatalog deutscher Bibliotheken, jetzt VK92 auf. Er befindet sich auf dem Stand von Ende 1991 und gibt zu 10 Millionen Titeln 19 Millionen Bestandsnachweise für 63 Bibliotheken. Von besonderem Gewicht ist die Einführung des Online-Ordering auch bei dieser Datenbank, das zur Zeit bei fünf Bibliotheken möglich ist. Soweit sich die Bestellung auf einen Auszug, etwa aus einem Kommentar, beschränkt, kann die Lieferung

Automatisierung der Einwabl mit Hilfe von Scripts

Die Datenbanken des DBI 

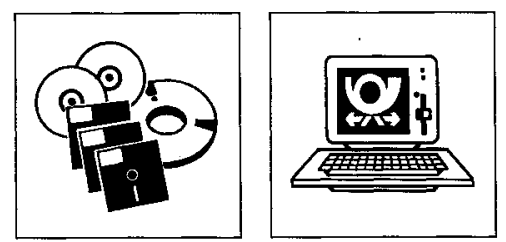

dabei per Fax erfolgen. Eines Hinweises wert ist schließlich, daß über das öffentliche Paßwort DBILINK (ohne Leerzeichen, ein Wort!) die Recherche (ohne Online-Ordering) in dieser Datenbank jedermann uneingeschränkt offensteht. Inhaltlich wurden einige Änderungen an der Feldstruktur der Datenbank und damit korrespondierend der Retrievalsprache durchgeführt, ohne aber Grundlegendes zu ändern. Die letzte Rechnermeldung ENTER BASE COMMAND fordert auf, eine der fünf Datenbanken des DBI zu wählen, die nunmehr kurz vorgestellt werden.

\section{ZDB: Zeitschriftendatenbank}

Ein überregionaler Katalog zum Nachweis von Zeitschriften und ähnlichen Publikationsmitteln (insgesamt ca 650.000 Titel) mit der Möglichkeit von Online Bestellungen. Diese Datenbank beinhaltet das umfassendste Angebot ihrer Art in der Welt.

NZN: Niedersächsischer Zeitschriftennachweis

Ein regionaler Katalog mit dem gleichen Inhalt wie die ZDB, in dem ca. 168.000 Titel nachgewiesen werdèn. Online Bestellungen sind nicht möglich.

ZBSB: Zeitschriftenkatalog der Bayerischen Staatsbibliothek (München) Ein lokaler Katalog (49.500 Titel) zum Nachweis von Zeitschriften und ähnlichen Publikationsmitteln.

GKS: Gesamtverzeichnis der Kongreßschriften

Ein überregionaler Katalog, in dem Veröffentlichungen von und zu Kongressen nachgewiesen sind. Die Titelaufnahme ist verhältnismäßig selektiv, so daß eine auch nur annähernde Vollständigkeit der Sammlung nicht erreicht wird.

VK: Verbundkatalog maschinenlesbarer Katalogdaten deutscher Bibliotheken

Ein überregionaler, den Bestand von 630 Bibliotheken umfassender Katalog, in dem ca 10.000.000 (Stand Ende 1991) Monographien und Dissertationen nachgewiesen werden. Soweit es nicht um Standortnachweise, sondern die sachliche Erschließung geht, ist im übrigen die nationalbibliographische Datenbank der Bundesrepublik Deutschland Bibliodata des Hosts STN der Aufgabenstellung nach zuständig, die alle deutschen Neuerscheinungen aller Wissensgebiete nachweist.

\section{Die ZDB-Zeitschriftendatenbank}

Anwahl

Mit dem Kommando BASE ZDB wird die Zeitschriftendatenbank ZDB gewählt.

ZDB: Überregionaler, elektronischer Nachweiskatalog

\section{Bedeutung}

Es handelt sich um einen überregionalen, elektronischen Katalog, in dem Zeitschriften, zeitschriftenartige Reihen, Serien und Zeitungen mit der Standortangabe der vorbandenen Bände, aber obne sachliche Erschließung einzelner Aüfsätze, nachgewiesen werden. Die Datenbank gibt demnach keine Auskunft über bestimmte Abhandlungen oder sonstige Texte, sondern ausschließlich über das Veröffentlichungsmedium, den 'papiernen Datenträger' sozusagen. Aufsätze und Gerichtsentscheidungen, etwa zu einer konkreten Rechtsfrage, muß der Benutzer vorher selbst ermitteln. Allerdings sind Informationen darüber, welche Bibliotheken die betreffenden Fachzeitschriften führen, oft unentbehrlich, um aus einer Fundstelle überhaupt Nutzen ziehen zu können. In einer solchen Lage ist beispielsweise ein Rechtsanwalt, der über juris einen Aufsatz gefunden hat, der in einer Fachzeitschrift veröffentlicht wurde, die in keiner der ihm zugänglichen Bibliotheken (Gerichts-, Universitätsbibliothek etc.) vorhanden ist. Solche Probleme werden in Zukunft dem Junisten immer mehr zu šchafffen machen. Ëinerseits düüfte die Ausdifferenzierung des Rechts mit der Entwicklung neuer Spezialgebiete und weiterer Fachzeitschriften als Folge hiervon fortschreiten und andererseits der finanzielle Spielraum der Bibliotheken, wenn überhaupt, nur noch begrenzt die Aufnahme zusätzlicher Titel zulassen.

Inhalt

Anzeige aller Standortnacbreise zu einem Zeitschriftentitel
Der Benutzer erhält als Ergebnis seiner Anfragen von der Datenbank die genauen bibliographischen Angaben zu den ihn interessierenden Zeitschriftentiteln mit allen Standortnachweisen, wobei er deren Ausführlichkeit selbst bestimmen kann. 
Der Datenausgabe muß selbstredend eine Suche vorausgehen. Sollen beispielsweise die Angaben zur Saarländischen Kommunalzeitschrift ermittelt werden, verläuft die Recherche folgendermaßen:
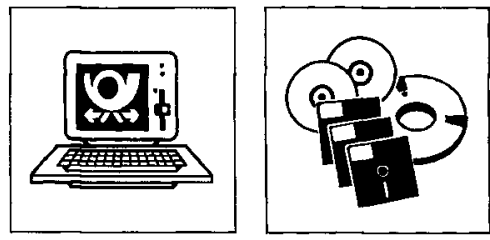

FIND SAARLÄNDISCHE KOMMUNALZEITSCHRIFT

1.00 NUMBER OF HITS IS 1

?S $\mathrm{F}=\mathrm{SUP}$.

1.00/000001 DBI: -ZDB /COPYRIGHT DBI/SBPK

TI: Saarländische Kommunal-Zeitschrift

CP: Hrsg.: Saarländischer Städte- und Gemeindetag und Landkreistag Saarland

$\mathrm{UT}$ : Mitteilungsblatt für Ratsmitglieder und Kommunalverwaltungen

$\mathrm{PP}$ : St. Ingbert

PU: Städẗe- u. Gemeindetag

$\mathrm{PD}: 8.1958-$

NO: Vorg. --> Mitteilungsblatt 206269

BER: la $\quad 15.1965$ - 18.1968: SIGN.: Zsn 32638

$\star \star \star E N D$ OF SHOW***

Der Inhalt des Downloadprotokolls ist weitgehend selbsterklärend. Mit dem Schlüsselwort FIND und dem Zeitschriftentitel wird der Befehl zur Suche erteilt, worauf das System die Profiltabelle (Sucbwortliste) mit einer laufenden Nummer für jeden Suchvorgang und der Zahl der Treffer ausgibt. Anschließend zeigt der Prompt „?" die Bereitschaft zur Entgegennahme des nächsten Kommandos an, hier S für SHOW (fast alle Befehle können mit dem ersten Buchstaben abgekürzt werden) zur Ausgabe der Dokumente. Das Ausgabeformat wurde mit $F=S U P$ auf den bibliograpbiscben Standard und die Bestände der sog. Lieferrantenbibliotbeken beschränkt, bei denen über den Rechner des DBI Online-Bestellungen möglich sind. Daneben existieren eine Vielzahl weiterer Ausgabeformate, die entsprechend den unterschiedlichen Interessen der sehr heterogenen, von Bibliothekaren bis $\mathrm{zu}$ interessierten Laien reichenden Benutzergruppen $\mathrm{zu}-$ sätzliche Felder berücksichtigen und/oder auf Standardfelder auch verzichten. Ähnlich wie bei juris mit GIB REGISTERNAME1 + REGISTERNAME2 + .....ist es schließ lich möglich durch $S F=F E L D N A M E 1, F E L D N A M E 2, \ldots$. das Ausgabeformat völlig in dividuell festzulegen. Die Ausgabe ist hier wegen des gewählten Formats und weil nur eine der Lieferantenbibliotheken die Zeitschrift führt, verhältnismäßig kurz, kann aber durchaus über mehrere Bildschirmseiten gehen, wobei mit $M O R E$ geblättert wird. END OF SHOW markiert das Ausgabeende.

Recherche-Möglichkeiten

Die Retrievalsprache (Abfragesprache) ist GRIPS, die dem von der Kommission der europäischen Gemeinschaften propagierten Standard für Datenbank-Abfragesprachen CCL folgt. Sie wurde von DIMDI entwickelt, einem Host mit vorwiegend medizinischen, aber auch rechtlich relevanten Informationen, zum Beispiel in der Datenbank ASYLDOC.

GRIPS kommt mit wenigen grundlegenden Kommandos aus, von denen die wichtigsten, nämlich BASE für die Wahl der Datenbank, FIND als Suchbefehl und SHOW als Ausgabeanweisung bereits vorgestellt wurden.

Ist der genaue Titel des gesuchten Aufsatzes (etwa nach einer juris-Recherche) bekannt, sind weitere Überlegungen zur (datenbanktechnischen) Formulierung der Suchfrage entbehrlich.

Andernfalls können die Suchfunktionen von GRIPS eingesetzt werden. So ist die Verknüpfung der - auch trunkierten - Stichwörter aus dem Titel, den Nebentiteln u.s.w. mittels der Buslscheni Operatoren ANTS, OR, NO' zulässig. Dabei lassen sich die Auswahl der Suchworte und deren Verknüpfung in einer Anweisung zusammenfassen.

Sehr vorteilhaft sind dabei als besondere Funktion von GRIPS die sog. Kontextoperatoren, welche die Position des Suchbegriffs in einem Datenfeld bestimmen (sog. Abstandssuche). Sie sind allerdings immer nur auf ein Datenfeld bezogen. Außerdem ist diese Funktion auf den sog. Freitext (die den Titel beschreibenden Felder), beschränkt. Bei der Suche mit sog. speziellen Suchfeldern wie CA (Körperschaften), SS (ISSN), CO (CODE) u.s.w. scheidet diese Möglichkeit aus. Allerdings kann ein Titel über einige dieser Register, insbesondere das Register $S S$ (für ISSN = internationale Standardnummer für fortlaufende Sammelwerke) meist sehr schnell und sicher gefunden werden.

Retrievalsprache nach CCL-'Standard' der EG

\section{Suchfunktionen}

Besonderes Feature: Abstandssuche 
Die Anzeige der sog. Profiltabelle erfolgt mit TAB. Die einzelnen Einträge der Profiltabelle können in der gleichen Weise wie die von Ihnen repräsentierten Suchbegriffe bei der Recherche verwendet werden.

Welche Suchbegriffe überhaupt in der Datenbank vorkommen, kann mit dem Kommando DISPLAY REGISTERNAME festgestellt werden. Es dient zur Anzeige eines tabellarischen Auszugs der Einträge des jeweiligen Registers. Die Übernahme eines oder mehrerer dieser Einträge in eine Suchanfrage wird durch die Nennung des/der auf der linken Seite der Tabelle erscheinenden Ordnungsziffer/n bewerkstelligt.

Allerdings dürften für den größten Teil der Datenbanknutzung Fragen der Suchtechnik eher von geringer Bedeutung sein. Anders als bei den der sachlichen Erschließung dienenden Datenbanken ist bei der ZDB als einem reinen bibliographischen Nachweissystem, wie schon erwähnt, die Suche nach bestimmten Aufsätzen nicht möglich, sondern nur das Veröffentlichungsmedium selbst recherchierbar, mit dessen normalerweise bekanntem Namen sich meist allein die interessierenden Detail-Angaben problemlos ermitteln lassen.

\section{Einzelheiten}

Der an Einzelheiten der Abfragesprache GRIPS interessierte Leser sei auf das ausführliche von dem Host DIMDI herausgegebene Benutzerhandbuch verwiesen, das neben einer genauen Beschreibung der "Grammatik" auch allgemeine Probleme der Abfragetechnik behandelt. Eine knappe Darstellung der Kommandos bietet auch die bereits erwähnte Kurzanleitung des DBI. Ihr kann neben weiteren Informationen zu den Datenbanken zusätzlich eine Übersicht der Such-und Ausgabefelder der ZDB entnommen werden. Ein registrierter Datenbankteilnehmer erhält im übrigen von dem DBI ein ausführliches Manual, das auch Fragen des Retrievals behandelt.

\section{Bestellung von Zielinformationen}

\section{Bedeutung}

Online-Bestellungen Bedeutung für die juristische Praxis
Die größte Bedeutung jedenfalls für den normalen Nutzer liegt in der Möglichkeit von Online-Bestellungen, die sowohl im Anschluß an eine Recherche als auch unabhängig hiervon möglich sind und zwar in Form einer Normal- oder Eilbestellung. Aus einer Recherche heraus wird mit dem Befehl ORDER eine Bestellung eingeleitet. Sie gewährt dem Anwender den Zugriff auch auf ihm sonst fast unerreichbare Dokumente, notfalls innerhalb eines Tages.

\section{Eine typische Anwendungssituation}

Welche Vorteile dieses "Zeitmoment” etwa in der Prozeßführung bringen kann, zeigt das folgende fiktive, aber dennoch realitätsnahe Beispiel:

Ein in Bad Neuenahr niedergelassener Anwalt wird am 31.03.1992 von einem dort ansässigen Schausteller, dem eine saarländische Gemeinde für ein am 03.04.1992 stattfindendes Volksfest einen Standplatz verweigert hat, konsultiert. In der Begründung des Bescheids weist die Gemeinde unter anderem darauf hin, sie habe hierbei die obergerichtliche Rechtsprechung beachtet, insbesondere die Grundsätze zur Verteilung des zur Verfügung stehenden Raums, die das für sie zuständige OV.G des Saarlandes in seinem Beschluß vom 26.09.1991 Az 1 W 103/91 aufgestellt habe, der in der Saarländischen Kommunalzeitschrift 1992 S. 16-18 veröffentlicht sei. Der Schausteller erteilt Mandat, den Standplatz vor Gericht zu erstreiten, sofern die Sache erfolgversprechend sei. Klar ist, daß nur eine schnellstmöglich zu beantragende einstweilige Anordnung dem Mandanten weiterhelfen kann. Die besondere Eilbedürftigkeit entbindet den Anwalt aber nicht von einer sorgfältigen Überprüfung der Rechtslage, wozu hier vor allem die Rechtsprechung des über eine einstweilige Anordnung in letzter Instanz entscheidenden OVG des Saarlandes gehört. Es ist eherr ùnwahrscheinlich, daß ein Rechtsanwalt in Bad Neuenahr direkten Zugriff auf die Saarländischen Kommunalzeitschrift hat. Bis zum nächsten Tag auf postalischem Weg eine Ablichtung bei dem OVG zu besorgen, dürfte zumindest problematisch sein. Eine Online-Bestellung der erwähnten Entscheidung als Telefax hilft hier weiter.

Ablauf einer Bestellung

Den Ablauf der Bestellung gibt das auf der nächsten Seite abgedruckte Download-Protokoll wieder. 
?ORDER

SELECTING FOR RECORD $=1$

$001: 1 \mathrm{~A} \quad 15.1965$ - 18.1968 : SIGN.: Zsn 32638

PLEASE SELECT BY NUMBER IN DESIRED SEQUENCE:

1

PLEASE ENTER THE RESPECTIVE LIBRARY IDENTIFICATION

SUPPLIER SUPPLIER USER IDENTIFICATION

$1 \mathrm{~A}$

$1 \mathrm{~A}$

LABEL FOR ADDRESS $=$ NO

UNTIL $\quad=28.04 .92$

01.04 .92

URGENT

YES

FORMAT $\quad=$ HARDCOPY

TELEFAX, Nr, $0681 / 324576$

REMARK

Dokument wird für Prüfung eines Antrags auf vorläufigen

Rechtsschutz benötigt, der spätestens am 01.04 .92 gestellt

sein muß.

PROMPTING FOR RECORD $=1$

$\mathrm{AU}$

Oberverwaltungsgericht des saarlandes

TI

Beschluss vom 26.09.1991 - 1 W 103/91

VOL

1992

PAGE

$16-18$

ORDER=4 PROCESSED; QN $=1$

Nach dem Bestellkommando ORDER übernimmt das System zunächst den Zeitschriftentitel sowie die übrigen bestellrelevanten Daten, nennt die möglichen Lieferbibliotheken und fordert zur Auswahl des Lieferanten durch die Angabe der entsprechenden Zeilennummer auf. Nach Eingabe einer eventuellen Supplier-Identification (Kundennummer des Lieferanten) werden die jeweils mit Standardangaben vorbelegte Art der Bestellung und die Lieferfrist erfragt und schließlich die bibliographischen Angaben zu dem Dokument selbst gemacht. Damit ist die Bestellung abgeschlossen, was mit der Systemmeldung OR$D E R=B E S T E L L N U M M \bar{M} \bar{R} \bar{P} \bar{O} \bar{C} E S S E D ; Q N=1$ bestätigt wird.

Ohne vorangehende Recherche wird eine Bestellung mit ORDER DIREKT eingeleitet. Allerdings muß der Benutzer nunmehr selbst die bibliographischen Angaben zur Zeitschrift und der Lieferantenbibliothek eingeben.

Die Bestellung wird in einer Datei gespeichert, welche die Lieferanten (= Bibliotheken) - mindestens einmal täglich lesen. Sie ermöglicht auch einen Überblick des Bearbeitungsstands der Bestellung mit INFO ORDER. 

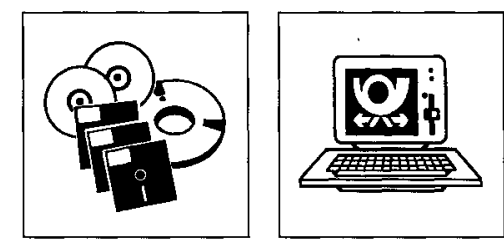

?INEO ORDER

\begin{tabular}{cccccc} 
ORDER NUMBER & SUPPLIER & QN DATE & UNTIL & STATUS & URGENT RECEIVED \\
\hline 4 & TRAIN & 131.03 .92 & 01.04 .92 & ORDERED & YES
\end{tabular}

Da der Verfasser nur als Testteilnehmer Zugang zur Datenbank hatte, war lediglich die Simulation einer Bestellung mit TRAIN als imaginären SUPPLIER (Lieferant) möglich.

Die Überprüfung des Inhalts der Bestellung selbst erfolgt mit $S H O W_{i} O R D E R$ :

?SHOW ORDER

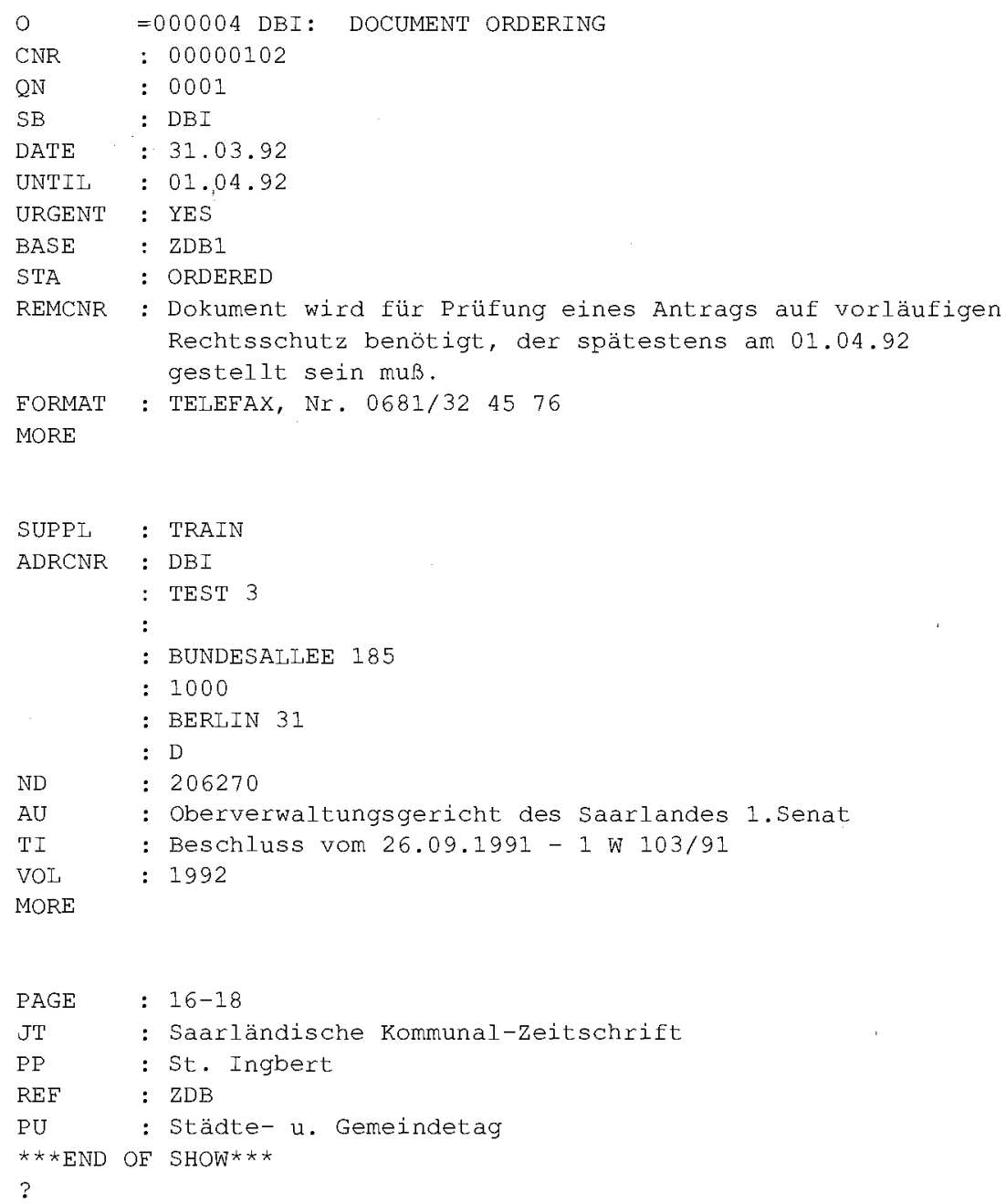

Bei einer nur simulierten Bestellung, wie im vorliegenden Fall, wird als Besteller grundsätzlich das DBI selbst genannt.

Dialogbeendigung

Das Kommando zur Beendigung einer Sitzung lautet STOP.

\section{Bilanz}

Bei insgesamt 650.000 Nachweisen zu Zeitschriften wird es eine Seltenheit sein, daß ein Jürist, ob Praktiker oder Wissenschaftler, einmal eine dort nicht erfaßte Zeitschrift suchen sollte. Regelmäßig dürfte auch eine Online-Bestellung über eine der acht Lieferantenbibliotheken realisierbar sein. Der elektronische Bestellvorgang ist verhältnismäßig einfach und läßt sich außerdem, entsprechende Kommunikationssoftware auf Anwenderseite vorausgesetzt, vollständig automatisieren.

Wïnschenswert: Erweiterung um Dateitransfer
Eine wesentliche, bereits jetzt prinzipiell realisierbare Verbesserung wäre die Erweiterung des Bestellsystems (natürlich im Zusammenwirken mit den angeschlossenen Bibliotheken) um die Möglichkeit, die gewünschten Dokumente als Datei zu empfangen. In diesem Falle könnte die Lieferung, weil ohne "mechanische" Zwischenschritte, praktisch" sofort, in 
Sekundenschnelle erfolgen und das Ergebnis ohne Umwege in den eigenen Rechner des Bestellers, etwa zur Bearbeitung in der Textverarbeitung, übernommen werden. Berücksichtigt man die immer größere Bedeutung von Publikationen in elektronischer Form und die (meist schon bei ihrer Erstellung) Erfassung auch von „geborenen” Druckerzeugnissen auf elektronischen Datenträgern, ist eine solche Funktion jedenfalls das Gebot der Zukunft, welches die Bedeutung der Trennung zwischen Recherche und Dokumentenbeschaffung im Interesse des Benutzers vermindert. Die damit zusammenhängenden urheberrechtlichen Probleme müßten lösbar sein. Auch die EG mißt der Lieferung von Dokumenten in elektronischer Form eine große Bedeutung zu und hat sie zum Gegenstand eines Projekts gemacht.

Insgesamt erleichtert aber schon heute die Zeitschriftendatenbank des Deutschen Bibliotheksinstitut für den Bereich der Zeitschriftenliteratur den Übergang von der Suche nach Informationen zu deren Beschaffung erheblich. Allerdings bleiben als Alternative Datenbanken von einzelnen Bibliotheken, die nach ihrem Aufbau und Zuschnitt den Interessen des jeweiligen Anwenders besser entsprechen können. Die ZDB des DBI hat aber den oft entscheidenden Vorteil, die größte Zahl an Zeitschriftentiteln nachzuweisen und die elektronische Bestellung bei acht großen Bibliotheken anzubieten.

jurpc.zip - jurpc.zip - jurpc.zip - jurpc.zip - jurpc.zip - jurpc.zip - jurpc.zip

\section{Deskjur - Eine Benutzeroberfläche, nicht nur für Juristen}

\section{Jörg H. Blankenburg, Dieter van Luit, Manfred Weibermüller}

DESKJUR ist ein Programm, welches dem Benutzer eines Computers das Starten von anderen Programmen erleichtern soll, indem es die Befehle, die er sonst von Hand an das Betriebssystem geben müßte, unter einem einzigen Kommando zusammenfaßt.

Entstanden ist DESKJUR unter der Leitung von Prof. Dr. jur. Dr. rer. nat. Herbert Fiedler aus den Arbeitszusammenhängen seiner Forschungsstelle für juristische Informatik und Automation an der Universität Bonn und seiner Arbeitsgruppe Informationsrecht bei der GMD.

Man nennt ein Programm wie DESKJUR eine Benutzeroberfläche oder einfach Oberfläche. Es ist möglich, die Oberfläche direkt beim Start des Computers aufrufen zu lassen, so daß der Benutzer nicht in direkten Kontakt zum Betriebssystem treten muß.

Seit einigen Jahren existiert der von der Firma IBM entworfene SAA-Standard (SystemAnwendungs-Architektur), der unter anderem auch eine Form festlegt, wie sich Programme, also auch Oberflächen, am Bildschirm präsentieren sollten, wenn sie dem SAAStandard genügen wollen. Immer mehr Programme sind inzwischen auf diese Weise zu bedienen, so daß der Benutzer nicht mehr bei einem Programm eine Funktionstaste, bei einem anderen eine Zahl, beim dritten einen Buchstaben und beim vierten eine Ctrl-Tastenkombination eingeben muß, um einen Befehl auszuführen oder einen Menüpunkt zu erreichen, sondern alle „verhalten” sich nach (fast) gleichen Regeln.

DESKJUR versucht, sich möglichst an diesen Standard zu halten.

DESKJUR ist mit Maus oder Tastatur bedienbar und arbeitet im Textmodus. DESKJUR

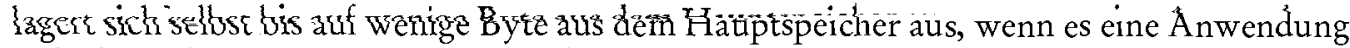
aufruft. Dadurch ist sichergestellt, daß alle Anwendungen, die unter DOS ohne DESKJUR laufen, auch unter DESKJUR funktionieren. Außerdem überläßt DESKJUR den aufgerufenen Programmen sämtliche System-Ressourcen, so daß es zu keinen Konflikten zwischen den aufgerufenen Programmen und DESKJUR kommen kann.

DESKJUR ist auf IBM-kompatiblen Computern lauffähig, die unter den Betriebssystemen MS-DOS, PC-DOS, DR DOS ab Version 3.30 arbeiten. DESKJUR benötigt maximal 250.000 Byte Platz auf der Festplatte und für jeden weiteren Benutzer nur einen Bruchteil davon (um 60.000 Byte).

Weitere Erläuterungen zum Programm (Installation etc.) finden sich in der Erläuterungsdatei auf der Diskettenbeilage für Abonnenten.

DESKJUR liegt dieser Ausgabe für Abonnenten bei.

SAA-Standard

Bedienwng mit Maus oder Tastatur 